17. HipPf.L, E. von-Prognosis of uveal sarcoma. Arch. f. Ophthal., Vol. CXXIV, pp. 206-220, 1930.

18. Continuation of statistical report on sarcoma patients. Arch. $f$. Ophthal., Vol. CXXIX, pp. 552-568, 1933.

19. Continuation of statistical report on sarcoma patients. Arch. f. Ophthal., Vol. CXXXV, pp. 76-78, 1936.

20. A new way of evaluating prognosis of uveal sarcoma. Arch. $f$. Ophthal., Vol. CXXXV, pp. 79-86, 1936.

21. JAENSCH, P - Prognosis of enucleation of melanosarcoma. Klin. Monatsbl. f. Augenheilk, Vol. LXXXIV, pp. 649-662, 1930.

22. KING, E. F - Pathogenesis of melanoma. Trans. Ophthal. Soc. U.K., Vol. LVII, Part I, pp. 97-106, 1937

23. MCKEE, S. H.-Primary sarcoma uveal tract, analysis of 27 cases. Canadian Med. Assoc., Vol. XXXVI, p. 361, 1936.

24. Morax, V.-Diagnosis and prognosis of melanotic tumours. Buli. et Mem. de la Soc. Franc. Ophtal., Vol. XLIV, p. 365, 1931.

25. MUIR, Sir RobERT.-Text book of pathology.

26. PARSONS, SIR J.-Diseases of the eye, 1936 edition.

27. RENARD, G.- Intra-ocular malignant tumours. Le Bulletin Médicale Librarie Arnette, Vol. LI, p. 368, 1937.

28. Samuels, B.-Study of anatomic and clinical manifestations of necrosis in 84 cases of sarcoma. Trans. Ophthal. Soc. U K., Vol. LIII, pp. 520-570, 1933.

29. Scales, J. L.-Melanosarcoma. New Orleans Med. and Surg. Ji., Vol. LXXXIX, p. 567, 1937.

30. Schovanic, B.-Intra-ocular sarcoma and its prognosis. Casopis Lekaru Ceskych Vladislavova Ulice, Vol. LXXVI, p. 920, 1937.

31. Seidel E.-Biology of sarcoma. Ber. ubr. die Versamm. d. deutsch., Ophthal., Vol. LI, p. 459, 1936.

32. Stallard, H. B.-Chapter on choroid in "The eye and its diseases" by Berens.

33. Teraskeli, H.-Prcgnosis of malignant tumours. Acta Ophthal., Vol. VI, p. 110,1928 .

34. TERRY, T. L. and JoHNS, J. P.-Sarcoma-malignant melanomas, statistical study of 94 cases. Amer. Jl. of Ophthal., Vol. XVIII, p. 903, 1935.

35. WILDER, H. C.-An improved technique for silver impregnation of reticulin fibres. Amer. Jl. of Path., Vol. XI, p. 817, 1935.

36. Wilder, H. C. and Callander, G. R.-See No. 4 above.

\title{
VITAMIN "C" (ASCORBIC ACID)-ITS THERAPEUTIC VALUE IN INFLAMMATORY CONDITIONS OF THE CORNEA
}

\author{
BY \\ Wing-Commander T. Keith LyLE, R.A.F.V.R. \\ and \\ Squadron-Leader D. W. McLean, R.A.F.V.R.
}

ALTHOUGH during the past six years a certain amount of research work has been carried out upon the somewhat doubtful relationship of vitamin " C" to the development of cataract, little attention has been given to the therapeutic value of this vitamin in certain other ocular diseases, namely, inflammation and ulceration of the cornea.

It has been held that the administration of vitamin " $\mathrm{C}$ " to patients suffering from certain types of cataract has met with some slight improvement in the visual acuity. ${ }^{1}$ Further, it has been 
shown that the cataractous lens contains a decreased amount of ascorbic acid, ${ }^{2}$ and that in a case of cataract the level of ascorbic acid is usually low in the urine, although it is normal in the blood.

It has been suggested, however, that the low ascorbic acid content of the lens might be the result of the cataractous changes rather than the underlying cause, and that the improvement of the vision noted in certain instances on treatment with ascorbic acid might be due to its effect on the media of the eye, the retinal vessels and the optic nerve. ${ }^{3}$

Experimental work on the effects of liquid mustard gas on the eyes of rabbits by Livingston and Walker ${ }^{4}$ showed that ascorbic acid given intravenously was of value in the treatment of the corneal lesions. The results of further work on this subject by Ida Mann and Pullinger ${ }^{5}$ showed, however, that from their experiments the value of ascorbic acid in counteracting the effects of gas keratitis was not apparent.

It was at the suggestion of Group Captain Livingston that the authors of this paper, working independently at two separate R.A.F. hospitals, have used large doses of ascorbic acid in the treatment of inflammatory conditions of the cornea.

Ascorbic acid is known to exist in high concentration in the lens and cornea of the human subject. It is a substance belonging to the group known as "redox potentials," which maintains a fine balance between the processes of reduction and oxidation, by acting either as a reducing or oxidising agent, whichever action is called for. It is therefore suggested that by introducing excess of ascorbic acid into the system the metabolism of the cornea might be accelerated, and in consequence the healing of inflammatory conditions of that structure hastened.

We have used ascorbic acid in the treatment of all cases in which severe inflammatory conditions of the cornea have necessitated inpatient hospital trealment, since the beginning of October, 1940.

The following records give details of each case.

It will be observed that in all instances the usual local treatment was given in addition to the injections of ascorbic acid, and also that in most cases local treatment alone was carried out over a period of time before using ascorbic acid.

CASE 1. Corporal F. W. T., aged 35 years-Mechanic. Dendritic corneal ulcer. On October 7 , he reported sick complaining of an inflamed right eye, which he had first noticed on the previous day. He was treated with boric lotion at his sick quarters. When seen by one of us two weeks later he had a large dendritic ulcer of the cornea of the right eye. It occupied the central part of the cornea, with branches extending upwards and downwards. The vision of his right eye was $6 / 24$. The left eye was normal, and the vision $6 / 5$.

$\mathrm{He}$ was admitted to hospital at once, and the right eye was 
treated with ung. atropine 1 per cent., pad and bandage. A slight improvement occurred for a few days, but a relapse subsequently took place, the ulcer becoming deeper and more extensive. Ung. scarlet red was tried, but little improvement resulted.

Thorough investigation for possible septic foci was carried out, but nothing abnormal was discovered. The Wassermann reaction of the blood was negative.

The eye hecame irritable to atropine, so ung. hyoscine $\frac{1}{2}$ per cent. was used instead.

On November 28, the ulcer was cauterised with pure carbolic acid. Slight improvement occurred but was not maintained.

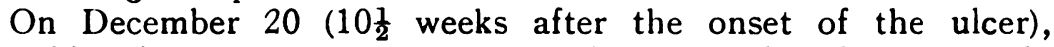
ascorbic acid treatment was commenced and was given intravenously as follows :-

$$
\begin{aligned}
& \text { December } 20 \quad \ldots \quad 250 \text { mgms. } \\
& \begin{array}{ccccc}
" & 22 & \ldots & " & , \\
" & 23 & \ldots & , & , \\
" & 24 & \ldots & 500 & , \\
" & 25 & \ldots & , & , \\
" & 26 & \ldots & " & , \\
, & 27 & \ldots & " & , \\
" & 30 & \ldots & , & , \\
" & 31 & \ldots & , & ,
\end{array}
\end{aligned}
$$

During this period the local treatment was continued. The progress made on the treatment with ascorbic acid was remarkable. The eye began to improve on the day following the first injection, and thereafter made steady progress. By December 31 (10 days after the first injection) the ulcer had completely healed, leaving only a faint superficial scar. Vision was $6 / 12$, and the eye was almost white.

Until his discharge from hospital on January 4, he was given ascorbic acid tablets ii t.d.s. (each tablet-250 mgms.). Up to date there has been no further ulceration, and the eye has remained quiet.

CASE 2. Flying Officer J. T., aged 24 years-Czech Officer. Disciform Keratitis. This officer was first seen towards the end of July 1940 when the left eye was irritable. There was small corneal infiltration, and the vision was 6/18. The inflammation had commenced when he was in France three or four weeks previously, and gutt. argyrol had been prescribed. Treatment with lot. ac. boric and gutt. atropine 1 per cent. was instituted, but the condition deteriorated gradually, and he was admitted to hospital on August 15, 1940. The right eye was normal.

There was then pronounced ciliary injection with a large infiltration of the cornea containing a round dense nebula. No focal 
sepsis was discovered. The B.S.R. was normal and the Meinicke reaction was negative. In spite of several forms of iocal treatment, the eye became steadily worse and presented a clinical picture of disciform keratitis, the vision being reduced to finger counting at one metre.

On October 22, 1940 he was given $500 \mathrm{mgs}$. of ascorbic acid intravenously, and this was repeated at intervals of 48 hours until November 1, 1940. In all he was given 3 grammes of ascorbic acid. Two days after the initial injection the eye was less irritable. By November 2, 1940 the eye was quiet and free from pain, though there was little change in the condition of the cornea. The improvement was maintained and the cornea cleared, at first slowly, then more rapidly. On discharge from hospital on December 3, 1940 the eye was white, the cornea showed only a thin paracentral nebula with a slightly denser centre $1 \mathrm{~mm}$. in diameter, and the vision was $6 / 6$. Re-examination two months later showed that the eye was still quiet.

CASE 3. Leading Aircraftsman M. K., aged 25 years.-Polish Airman. Scleritis with early sclerosing keratitis. Admitted to hospital October 11,1940, The left eye had been inflamed for approximately six months, and there had been previous similar attacks over a period of three years.

There was considerable conjunctival injection with a slightly raised area of dull purplish colour over the ciliary region at two. o'clock and a smaller one at seven o'clock. The deep vessels in these regions showed considerable engorgement. The cornea showed an opacity of moderate density towards the temporal side of the pupillary area, and three white superficial patches of sclerosis spreading from the limbus in the neighbourhood of the larger scleritic focus. The eye was amblyopic but showed a normal fundus. Meinicke's reaction was negative. The right eye was normal.

Local treatment was instituted with boric lotion and ung. atropine with hyd. oxid. flav. a.a. 1 per cent. sodium salicylate was given by the mouth. Two courses of short wave diathermy were given, the first being followed by temporary amelioration, but with that exception there was no improvement. Ascorbic acid was first given (500 mgs. intravenously) on November 13, 1940 and this repeated on alternate days up to November 21, 1940. During these eight days the inflammation subsided rapidly, the pain ceased and the cornea became clearer.

The improvement was maintained up to December 10, 1940 when there was a return of redness and irritability of the eye, which increased for three days. Five further injections. were given between December 14 and December 21, 1940 resulting in improvement. After this treatment the eye remained quiet, and on the patient's discharge to his Unit on January 4, 1941, it was white 
except for dull discolouration at the sites of scleritis. Examination with the slit-lamp revealed one faint paracentral nebula, apart from which the cornea was clear. Altogether 5 grammes of ascorbic acid were administered. He was re-examined on March 18, 1941, and it was reported that the eye was still quiet.

CASE 4. Aircraftsman R. D., aged 29 years. Superficial punctate keratitis. Admitted to hospital December 12, 1940. The right eye had been painful and inflamed for six weeks. Boric irrigations had been employed at his station sick quarters, but no improvement had resulted.

Six small superficial infiltrations were present in the lower half of the cornea, and there was moderate circum-corneal and conjunctival injection, the lower fornix being especially velvety. The patient complained of some pain, and there was profuse lacrimation. A conjunctival swab gave no growth on culture. The Wassermann reaction was negative, and no septic focus was found. Treatment with gutt. atropine 1 per cent. and spoon-bathing was instituted, and four injections of ascorbic acid (2 grammes in all) were given between December 14 and December 18, 1940. After the second injection the eye showed great improvement and the lacrimation was less. By December 19, 1940 the eye was quiet and showed only faint corneal opacities which had disappeared by December 30 , 1940 , though the lower fornix was still slightly oedematous.

CASE 5. Aircraftsman H. R. V., aged 26 years. Post vaccinial keratitis. Admitted to hospital January 13,1941. Three weeks earlier he had been vaccinated, and the wound on his arm had become infected. This was followed by pain and inflammation of the left eye. He had been under treatment at his station hospital from January 4, 1941.

On admission, the left eye showed pronounced ciliary injection. There was a band-shaped infiltration $5 \mathrm{~mm}$. long by $2 \mathrm{~mm}$. wide in the upper part of the cornea. The iris appeared slightly discoloured, and the anterior chamber deep. Pain and lacrimation were prominent. On the left upper arm was a healing ulcer.

A conjunctival swab gave no growth on culture. The vitamin C content of the urine was $0.4 \mathrm{mg}$. per cent. (increased after treatment). Local treatment consisted of irrigations, atropine 1 per cent. drops and spoon-bathing. No appreciable improvement resulted. Injections of ascorbic acid were started on January 20 , 1941. On January 24, 1941 the eye was considerably quieter, and this improvement was maintained. By February 10, 1941 the eye was quiet with neither pain nor lacrimation. On discharge on February 17, 1941 the eye was white and there was no trace of corneal scarring. The arm ulcer had healed. He was given 2.5 grammes ascorbic acid in five injections.

CASE 6. Aircraftsman D. S. W., aged 26 years. Phlyctenular 
keratitis. Admitted January 27, 1941. His left eye had been inflamed for three months, treatment having been carried out at his station sick quarters with irrigations and atropine.

The pupil was semi-dilated and slightly active; there was moderate conjunctival and ciliary injection; and the cornea showed several diffuse superficial infiltrations, phlyctenular in type, but without vascularisation. The vitamin $\mathrm{C}$ content of the urine was $0.7 \mathrm{mg}$. per cent. which did not increase. A conjunctival swab provided a few colonies of staphylococcus albus. The local treatment was continued, and 2 grammes of ascorbic acid administered in four injections.

The eye settled down rapidly. The infiltrations faded, and when the patient was discharged on February 19, 1941, they were barely perceptible.

CASE 7. Aircraftsman T. F. B., aged 22 years. Superficial punctate keratitis. On February 3, 1941, the left eye was injured by a twig. It became red and painful two days later. $\mathrm{He}$ was admitted to hospital on March 14,1941. The left eye showed much conjunctival and ciliary injection with a group of small subepithelial infiltrations of the cornea. There was blepharospasm and copious lacrimation. The vitamin $\mathrm{C}$ content of the urine was 0.7 mg. per cent.

Treatment with boric irrigations, gutt. atropine 1 per cent., and dry heat was carried out.

Ascorbic acid treatment was commenced immediately, and on February 19, 1941, the eye was already more comfortable and less red. By February 23, 1941, it was quiet, and the cornea almost clear, although the lacrimation and blepharospasm persisted. On March 3, 1941, the patient developed a catarrhal cold, and the eye became more irritable. The original infiltrations had disappeared entirely, but a new one developed in the pupillary area, becoming acute and breaking through the epithelium (demonstrated by staining with fluorescein). $\mathrm{He}$ is still under treatment with ascorbic acid, and the eye is gradually settling down. Up to the time of recording, he has received 5 grammes of ascorbic acid. His excretion of vitamin $\mathrm{C}$ increased after each injection.

CASE 8. Flight-Sergeant R. N. T., aged 35 years. Phlyctenular "ring" ulcer. His right eye became inflamed and painful on February 15, 1941, while he had a cold. There was no history of trauma. He was treated at his unit hospital until February 21, 1941, when he was sent in to the regional hospital.

On admission, there was intense inflammation of the conjunctiva, both bulbar and palpebral, with pronounced circum-corneal injection. The upper quarter of the cornea showed definite pannus. The remainder of the periphery was ulcerated in an unbroken line of yellowish slough about $2 \mathrm{~mm}$. broad. Over the rest of the cornea 
the epithelium was so oedematous and wrinkled as to be quite opaque. Pain was not a prominent feature, but there was much blepharospasm and lacrimation, and a muco-purulent discharge. A conjunctival swab showed staphylococcus albus. The vitamin $\mathrm{C}$ content of the urine was too small to estimate, though it increased greatly after treatment.

Local treatment was confined to irrigations with boric lotion, atropine drops and spoon bathing. $500 \mathrm{mg}$. ascorbic acid were injected intravenously every day from February 22, 1941, to March 3, 1941, and then on alternate days until March 15, 1941. In all, $7 \cdot 5$ grammes of ascorbic acid were given intravenously. After this treatment, tablets of ascorbic acid (250 mgms., "Roche") were administered orally at the rate of six per diem.

The condition of the cornea began to improve forty-eight hours after the first injection, and by March 1,1941, the oedema of the epithelium and stroma had subsided sufficiently to allow the iris to be seen. This was discoloured, but the pupil was well and evenly dilated. The ulcer lost its yellow edge and commenced to heal. On March 15, 1941, the eye was quiet, and the corneal epithelium was restored except for one facet on the temporal side. On March 20, 1941, the eye was almost white, and the cornea normal except for a little flattening of the epithelium at the periphery. The pannus had almost disappeared. Their was no pain, blepharospasm, lacrimation or conjunctival discharge.

CASE 9. Aircraftsman W. J. P.; aged 28 years. Superficial punctate keratitis. History of inflamed left eye for two months. He had been treated at his station sick quarters with boric lotion and atropine drops, with little improvement at first, but subsequently the condition gradually cleared up.

The right eye became inflamed four days ago. This was treated with boric lotion and atropine drops, but with no improvement. On admission, there was much conjunctival injection with photophobia and blepharospasm. The right cornea showed a typical superficial punctate keratitis.

Treatment was commenced with atropine drops and boric lotion. A slight improvement occurred.

On the fourth day, ascorbic acid injections were commenced, and the rapidity with which the condition cleared up was dramatic. 500 mgs. were given daily for four days, at the end of which time the eye was white. No recurrence has taken place over a period of several months.

CASE 10. Aircraftsman N. H., aged 26 years. Generalized seborrhoeic dermatitis, with keratitis and corneal ulceration of both eyes. History of seborrhoeic dermatitis of the scalp, commencing six weeks ago, with inflammation of the eyes for the last week. He had a diffuse seborrhoeic dermatitis of the skin, mainly involving 
the scalp; face and flexures, causing much irritation. Both eyes showed superficial ulcers with vascularisation. Blepharospasm and photophobia were severe, causing bilateral spastic entropion.

$\mathrm{He}$ was admitted to hospital, and his eyes treated with atropine and parolein drops. The skin of his trunk and face was treated with sodium bicarbonate lotion and calamine lotion. Little improvement occurred for the first four days on this line of treatment.

The ascorbic acid clearance test was then carried out, with the following results :-

First day. - $300 \mathrm{mgs}$. ascorbic acid given by mouth. Urine volume $=128$ c.c. during subsequent five hours. Vitamin " $\mathrm{C}$ " percentage $=3.8 \mathrm{mgs}$. per cent.

Second day. - $300 \mathrm{mgs}$. ascorbic acid given by mouth. Urine volume $=107$ c.c. during subsequent . five hours. Vitamin " $\mathrm{C}$ ". percentage $=3 \cdot 9$ mgs. per cent.

Third day. $-300 \mathrm{mgs}$. ascorbic acid given by mouth. Urine volume $=150$ c.c. during subsequent five hours. Vitamin " $\mathrm{C}$ " percentage $=5 \cdot 2 \mathrm{mgs}$. per cent.

These figures represent an initial ascorbic acid deficiency of 1,500 mgs. (Saturation is taken as corresponding with $5 \mathrm{mgs}$. per cent.)

On the following day, ascorbic acid injections were commenced, and given daily at the rate of $500 \mathrm{mgs}$. per injection for two weeks. The improvement in the eye condition after four injections was dramatic. Blepharospasm disappeared, and the corneae became much clearer.

Local treatment by means of hyoscine drops (owing to the fact that atropine irritation occurred) was continued, and at the end of two weeks the eyes had returned to normal, with the exception of two fine superficial corneal nebulae.

He is still under treatment for the skin condition, but the eyes are white and no longer irritable. Vision is $6 / 6$ each eye.

\section{Comments}

In cases 1 to 3 there were well established infective conditions with severe corneal involvement. In each case more orthodox treatment over a considerable period proved unavailing, but after injections of ascorbic acid there was a rapid and pronounced improvement. In Case 8 the eye was in a serious condition and appeared to be threatened with imminent perforation and/or panophthalmitis. In this instance, apart from the use of ascorbic acid, treatment was reduced to a minimum. This was regarded as a stringent test of the value of vitamin $\mathrm{C}$ injections, and in this case cure was both rapid and strikingly complete.

Case 7 is somewhat indeterminate. The immediate result was satisfactory, but a further infiltration developed, involving the deeper layers of the corneal stroma, and this proved much more resistant. 
Case 5 was a severe keratitis of somewhat unusual type, which resolved completely without any residual scarring. The disappearance of corneal opacities was also notable in Case 6, where the scars had both the history and the appearance of being of long standing. In both of these cases, as in Case 3 , a similar result might have been attained by more usual methods, but in all of them recovery appeared to be greatly accelerated.

In the first three cases, which were treated tentatively and without great expectation, no attempt was made to explore the output of vitamin C. Thereafter, the vitamin C content of the urine was estimated before and after treatment for the sake of comparison. Samples were collected from 30 patients in good general health, and on full diet, and titrated with a standard solution of dichlorophenolindophenol. It was found that the average content was 0.78 milligrammes per 100 cubic centimetres of urine. Estimations made before treatment varied considerably, and appeared to have little significance. During treatment three samples were taken daily from each patient, and an appreciable increase in the amount of vitamin C excreted was taken to indicate that there was no general deficiency. The rate of excretion appeared to be highest five or six hours after an injection.

In one instance only (Case 6) was there no increase in output.

We have tried ascorbic acid in the treatment of five cases of acute iritis, but with no beneficial effect. In one instance, which was of luetic origin with a strongly positive Wassermann reaction, the condition appeared to be aggravated by the injections as the following case record shows :-

Corporal J. G. G., aged 21 years. Acute irido-cyclitis.-In April, 1940, he had an inflammatory condition of the right eye which proved to be irido-cyclitis. The Wassermannn reaction was strongly positive, and the condition, which was treated elsewhere by local applications and anti-luetic measures, cleared up entirely in six months.

On December 26, 1940, the left eye became slightly red and irritable. He was admitted to hospital two days later. The left eye showed a slight degree of ciliary injection, and the anterior chamber was a little deeper than normal. The right eye showed no abnormality.

Treatment with gutt. atropine was commenced, and there was no change until January 10, 1941, when the left eye began to be more irritable and showed fine deposits on the posterior corneal surface. Frequent dry heat was added to the treatment.

The condition grew rapidly worse with the formation of many K.P., some being of the mutton-fat type. There was considerable pain in the eye.

The vitamin $C$ content of the urine was 0.9 mgms. per cent. 
Treatment with ascorbic acid was begun on january 25, 1941, and injections were given at frequent intervals up to February 14, 1941, when 3.5 grammes had been administered.

During this time, the state of the eye, with some fluctuations, deteriorated steadily, and on February 19, 1941, the hyperaemia of the iris was severe enough to give rise to an hyphaema. From that date it began to settle down, the hyphaema became absorbed, and the pain was much diminished.

Two further injections of ascorbic acid were given-on March 3, and March 5, 1941. On March 7, 1941 the eye was again very irritable with a recurrence of hyphaema. No further ascorbic acid was given, and the condition is now greatly improved, although numerous fine K.P. are still to be seen. Despite a further course of N.A.B., the Wassermann reaction is still strongly positive.

\section{Conclusions}

Although there is much further work to be done on the subject, from the limited number of cases treated we think the following conclusions may be drawn :-

1. In certain corneal conditions-notably ulceration, superficial type of keratitis and chronic corneal opacities - treatment by means of ascorbic acid intravenously is of therapeutic value. The improvement in most cases is almost dramatic.

2. In most cases treated, there is no reason to believe that a general vitamin "C" deficiency exists. It appears, therefore, that the beneficial results are obtained by flooding the bloodstream with excess of ascorbic acid.

3. Ascorbic acid appears to be of no value in the treatment of iritis. It may even have a detrimental effect in certain instances.

4. The injections used (ascorbic acid "Roche" concentrated vitamin " $\mathrm{C}$ " ampoules, $500 \mathrm{mgs}$. per injection) caused no local or general reaction.

5. Dosage:-We recommend the daily injection of $500 \mathrm{mgs}$. intravenously until active inflammation of the eye has ceased, preferably followed by ascorbic acid tablets given orally (Tabs. ii t.d.s., each tablet containing $250 \mathrm{mgms}$ ).

\section{BIBLIOGRAPHY}

1. Josephon, E. M. (1935).-Science, Vol. LXXXII, pp. 222-223.

2. Hawley, E. E. and Pearson, O. (1938).-Arch, of Ophthal., Chicago, Vol. XIX, p. 959.

3. Bouton, Jnr., S. M. (1939).-Arch. of Int. Med., Chicago, Vol. LXIII, p. 930.

4. Livingston, P. C. and Walker, H. M. (1940).-Brit. Jl. Ophthal., Vol. XXIV, No. 2.

5. Mann, Ida and Pullinger, B. D. (1940).-Brit. Jl. Ophthal., Vol. XXIV, No. 9. 